\title{
Cutaneous Histoplasmosis without Symptomatic Lung Involvement - A Series of Three Cases
}

Dipankar Pal", Parikshit Mullick, Dushyant Kumar Lahre, Avijit Das and Soumyadip Chatterji

School of Tropical Medicine, Kolkata, India

*Corresponding author: Dipankar Pal, RMO cum Clinical Tutor, Department of Tropical Medicine, School of Tropical Medicine, Kolkata, India, Phone: +91-9432113713; E-mail: dipankarpal.2009@gmail.com

Received date: May 15, 2015, Accepted date: May 20, 2015, Published date: May 23, 2015

Copyright: ( 2015 Dipankar $\mathrm{P}$ et al. This is an open-access article distributed under the terms of the Creative Commons Attribution License, which permits unrestricted use, distribution, and reproduction in any medium, provided the original author and source are credited.

\section{Medical Image}

Keywords: Cutaneous histoplasmosis; Immunocompetent; Immunocompromised subjects

\section{Case Discussion}

Histoplasmosis caused by Histoplasma capsulatum, a dimorphic fungus is endemic in America but also reported from different parts of the world including India. It preferentially causes disease in immunocompromised hosts in the form of acute as well as chronic lung involvement. Sometimes there is disseminated and progressive disease in immunosuppressed individuals with or without affecting CNS with high mortality rate.

Case 1: Immuno competent patient.

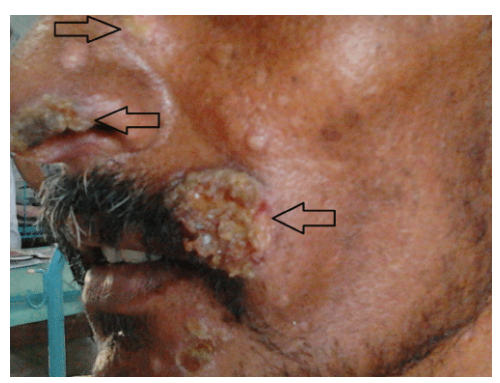

Figure 1: Lesions before treatment (arrow)

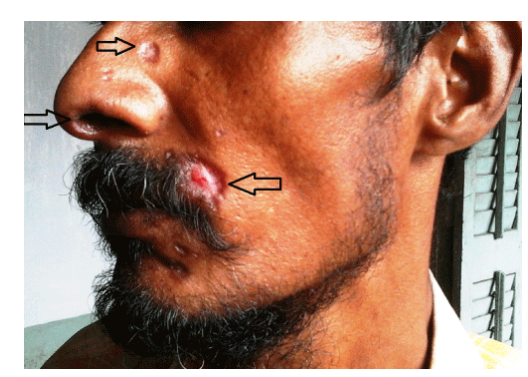

Figure 2: Lesions after treatment (arrow)

According to various literatures cutaneous histoplasmosis is less commonly seen than its pulmonary counterpart even in immunocompromised patients. In immunocompetent patients, it is rather a rare entity.
Case 2: Immunocompromised female on anti-retroviral therapy

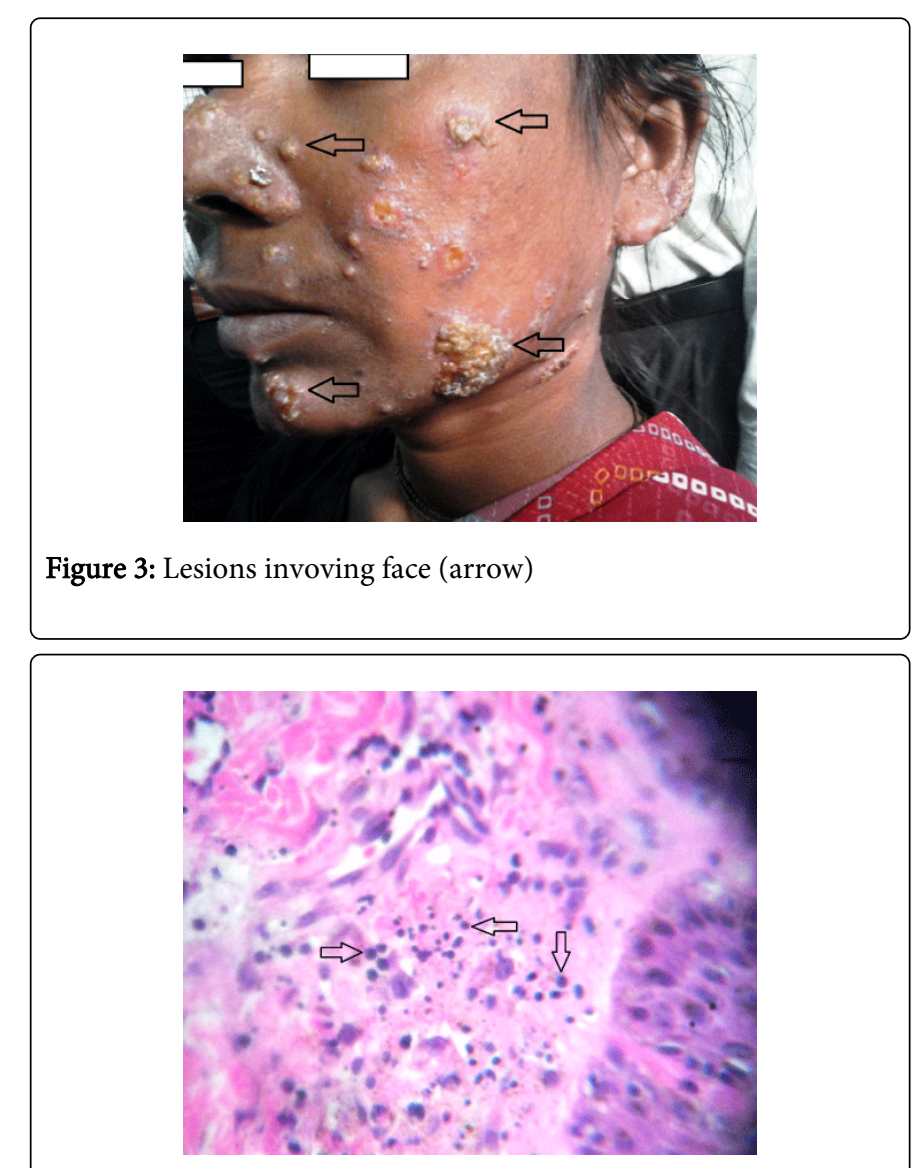

Figure 4: Skin biopsy showing the organism (arrow)

We came across three cases of cutaneous histoplasmosis in our institute; one is immunocompetent (Case 1) (Figure 1 and 2) and two of them are immunocompromised (HIV infected on anti-retrovirals with low CD4 count) (Case 2,3) (Figures 3-7).

None have chest symptoms at presentation with chest $\mathrm{x}$ rays essentially normal. Histoplasma is detected in skin biopsy in all three patients and successfully treated with amphotericin B.

Case 3: Immunocompromised patient on anti-retroviral therapy 
Citation: Dipankar P, Parikshit M, Dushyant K, Avijit D, Soumyadip C, et al. (2015) Cutaneous Histoplasmosis without Symptomatic Lung Involvement - A Series of Three Cases. Trop Med Surg 3: I101. doi:10.4172/2329-9088.1000I101

Page 2 of 2

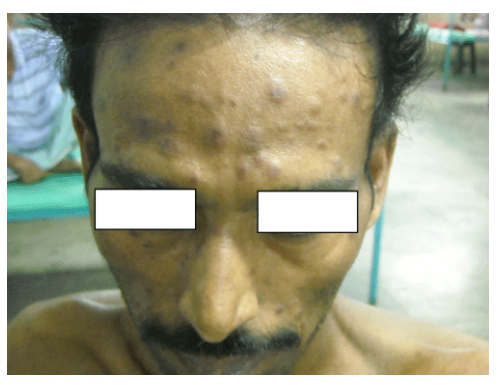

Figure 5: Lesions over whole face

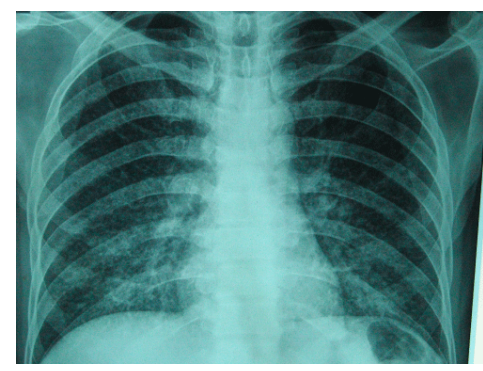

Figure 6: Normal Chest X ray

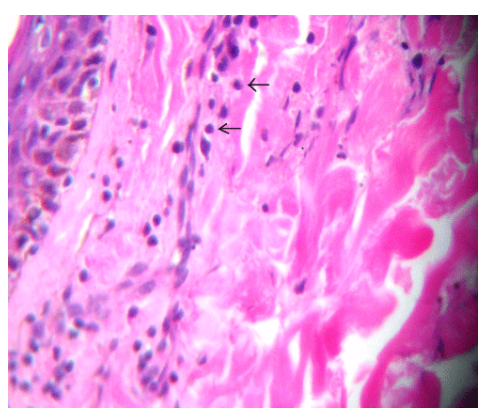

Figure 7: Skin biopsy showing organism (arrow)

\section{Key Clinical Message}

Histoplasma should be kept in mind along with blastomycosis, coccidioidomycosis, paracoccidioidomycosis, Penicillium marneffei, cryptococcosis with typical skin involvement both in endemic and non-endemic areas.

Conflict of interest: None declared 